Full Length Article

\title{
Low salinity effect on the recovery of oil trapped by nanopores: A molecular dynamics study
}

\author{
Chao Fang ${ }^{\mathrm{a}}$, Yafan Yang ${ }^{\mathrm{b}}$, Shuyu Sun ${ }^{\mathrm{b}}$, Rui Qiao ${ }^{\mathrm{a}, *}$ \\ ${ }^{a}$ Department of Mechanical Engineering, Virginia Tech, Blacksburg 24061, VA, USA \\ ${ }^{\mathrm{b}}$ Division of Physical Science and Engineering, King Abdullah University of Science and Technology, Thuwal 23955-6900, Saudi Arabia
}

\section{A R T I C L E I N F O}

\section{Keywords:}

Low salinity waterflooding

Thin brine films

Molecular dynamics

Osmosis

\begin{abstract}
A B S T R A C T
Low salinity waterflooding (LSW) is an effective method for enhancing the oil recovery from many reservoirs, and its success has been traced to a host of low salinity effects. In this work, we perform molecular dynamics simulations to study the feasibility of recovering oil trapped by nanopores by lowering the reservoir salinity. The oil is initially trapped by a slit nanopore, with a portion of the oil protruding from the pore entrance. After the reservoir salinity is lowered, the thin brine films that separate the oil and pore walls become thicker to drive some of the trapped oil out of the pore. We quantify the free energy profile of this process and clarify the underlying molecular mechanisms. Interestingly, the brine film growth is dominated by the water transport from the brine reservoir into the pore rather than by the depletion of ions from the brine film. These results provide molecular evidence that low salinity brines benefit the recovery of the oil trapped by nanopores. They highlight that when ion depletion from thin brine films is suppressed, the osmosis of water can play a fundamental role in the expansion of the brine films; thus, the enhanced oil recovery. The slow osmosis of water through thin brine films and thus the slow displacement of oil from the pore may help explain the anomalously slow oil recovery reported in micro-modeling experiments of LSW.
\end{abstract}

\section{Introduction}

Enhanced oil recovery has gained significant attention in meeting the growing energy demand as the average oil recovery factor from mature oil fields worldwide is $20 \%-40 \%$ in contrast to the typical recovery factor of $80 \%-90 \%$ from gas fields [1]. Low salinity waterflooding (LSW) is a promising technique for enhanced oil recovery that works by reducing/modifying the ionic content of the injected brines [2]. For instance, LSW in sandstone reservoirs is believed to enhance the oil recovery factor by $5-20 \%$ of the original oil in space [3]. Despite the growing interest and applications of LSW, the mechanism underlying LSW is still a topic of research and debate, in part because of the diverse composition of oil-brine-rock (OBR) systems and the complexity of LSW processes [4].

Altering the wettability of rock surfaces from a more oil-wet state to a more water-wet state is widely postulated as a major mechanism for LSW $[3,5,6]$. The wettability change has been confirmed by various experiments including chromatographic wettability tests, contact angle measurements, spontaneous imbibition tests, core flooding, etc [7]. Apart from these experimental works, the wettability alteration process has been modeled and captured by using empirical relations between wettability and salt solution parameters [8], taking into account surface reactions mechanistically [7], and focusing on the kinetics of oil detachments from model rock surfaces [9]. Although a complete understanding of the wettability alteration by LSW remains elusive at present, theories such as $\mathrm{pH}$ effect, multicomponent ionic exchange, and double layer expansion have been proposed, and many of them center on the modification of the thin brine films between oil and rock surfaces in OBR systems $[10,11]$. Some of these theories are supported by molecular dynamics (MD) simulations, e.g., the modification of the contact angle of oil droplets and the increase of the disjoining pressure driving the expansion of brine films between oil and rock surfaces [12-16].

The previous experiments and simulations, especially those on the wettability alteration, have improved our understanding of LSW greatly. However, a majority of these studies focused on oil droplets near flat rock substrates, whereas the highly porous structure of rock is often not accounted for. In oil reservoirs, however, a vast number of narrow pores exist. How LSW affects the recovery of the considerable amount of oil hold in these pores is little known. In this work, we perform MD simulations to study how the salinity reduction in brine reservoirs affects the recovery of oil trapped in nanopores. A key feature of our MD system is that the thin brine films sandwiched between the

\footnotetext{
* Corresponding author.

E-mail address: ruiqiao@vt.edu (R. Qiao).
} 


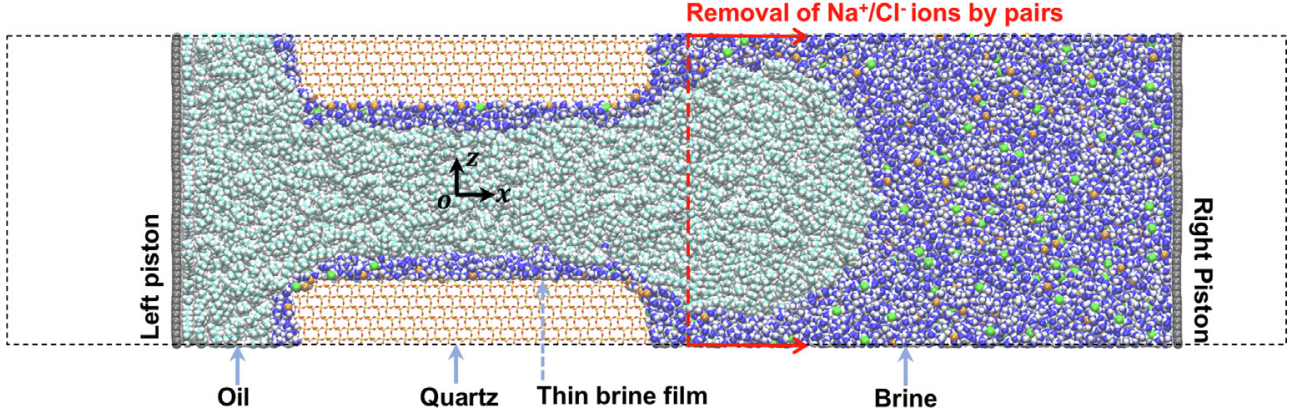

Fig. 1. The MD simulation system. The model system features $n$-decane oil trapped by a quartz nanopore and a brine reservoir. The left piston is fixed, and the pressure in the brine reservoir is regulated by the right piston. Initially the brine reservoir is filled with $2 \mathrm{M} \mathrm{NaCl}$ solution. The simulation box is marked using the dashed black lines, and the system is periodic in all three directions. At $t=0$, the salinity of the brine reservoir is lowered by removing all $\mathrm{Na}^{+}$and $\mathrm{Cl}^{-}$ions by pairs in the region to the right of the red dashed line $(1.5 \mathrm{~nm}$ away from the pore

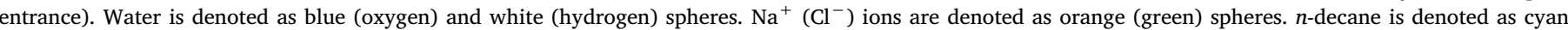

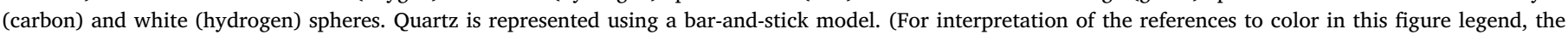
reader is referred to the web version of this article.)

trapped oil and rock surfaces are considered explicitly. The rest of the manuscript is organized as follows. In Section 2, the model system and simulation methods are presented. In Section 3, results such as the expansion of thin brine films and the displacement of oil from the pore are presented. The thermodynamics and dynamics of these processes and the underlying ion and water transport are discussed. Finally, conclusions are drawn in Section 4.

\section{Models and methods}

\subsection{Molecular systems}

Fig. 1 shows a snapshot of the MD system. The system consists of an oil reservoir, a slit rock nanopore, a brine reservoir, and the oil and brine in the nanopore. The oil and brine reservoirs are both bounded by a rigid piston. The piston facing the oil reservoir is fixed, and part of the oil in the nanopore protrudes out of the pore entrance as a droplet. Therefore, the oil in the reservoir and nanopores are trapped initially. The pressure of the brine reservoir is kept at $1 \mathrm{MPa}$ by applying an appropriate force on the piston facing it.

The rock is modeled as quartz, which is the major constituent of sandstones [10]. The oil is made of pure $n$-decane. Initially, the brine reservoir is filled with a $2 \mathrm{M} \mathrm{NaCl}$ solution. The high salinity used here is close to the salinity of the formation brine of some oil reservoirs [17]. The system is periodic in all three directions, but two large vacuum spaces are placed outside of the pistons to effectively eliminate the periodicity in the $x$-direction. The system measures 45.00, 3.93, and $9.68 \mathrm{~nm}$ in the $x, y$, and $z$-directions, separately. The rock pore is $\sim 11 \mathrm{~nm}$ long in the $x$-direction, and its width in the $z$-direction is $6 \mathrm{~nm}$ (see below). There are about 16,000 water molecules and 1400 decane molecules in each of the systems we studied.

\subsection{Molecular models}

The rigid SPC/E model is used for the water [18]. $n$-decane is modeled using the optimized parameter set developed from the original OPLS-AA force fields for linear alkanes [19]. The force fields parameters for $\mathrm{Na}^{+}$and $\mathrm{Cl}^{-}$ions are taken from Ref. [20]. The pore walls are cleaved from the $\left(\begin{array}{lll}1 & 0 & 1\end{array}\right)$ plane of a bulk $\alpha$-quartz crystal using the method described in Ref. [21]. The pore surfaces have a silanol group density of $5.92 \mathrm{~nm}^{-2}$ and are strongly hydrophilic. As such, a thin brine film forms between the oil (n-decane) and the quartz surface (see Fig. 1) as observed experimentally [22,23]. The zero plane of the quartz pore wall is defined as the z-position of the second outmost layer of surface silicate atoms. The nominal pore width, which is defined as the distance between the zero planes of the opposing pore surfaces, is $6 \mathrm{~nm}$. The surface of the quartz pore has a charge density of $-0.12 \mathrm{C} / \mathrm{m}^{2}$, consistent with the fact that quartz surfaces are negatively charged at most $\mathrm{pH}$ [24]. This surface charge density is obtained by the uniform deprotonation of the outmost surface silanol groups and then redistributing the net surface charge over the remaining surface atoms out of the quartz's zero plane [21]. The Lennard-Jones (LJ) potential and partial charges of quartz atoms are taken from the CLAYFF force fields $[21,25]$. All silicate and oxygen atoms of the quartz are fixed. The surface silanol groups' hydrogen atoms are allowed to move by considering their bonded interactions with other atoms of the surface silanol groups. The Lorentz-Berthelot combination rule is used to obtain the LJ parameters between dissimilar atoms.

\subsection{Simulation methods}

MD simulations are performed using the Gromacs 5.1.4 package [26]. The parallel simulations are performed using MPICH with 96 cores on 4 nodes. The bond and angle of water are constrained using the SETTLE algorithm. An NVT ensemble with the velocity rescale thermostat and a time constant of $1 \mathrm{ps}$ is implemented at $350 \mathrm{~K}$ [27], which is relevant to the reservoir conditions. A time step size of $2 \mathrm{fs}$ is used. The LJ potentials are calculated with a global cutoff of $1.2 \mathrm{~nm}$, and the long-range energy and pressure corrections are applied. The particle mesh Ewald method is used to calculate the electrostatic interactions (real-space cutoff: $1.2 \mathrm{~nm}$; FFT spacing: $0.12 \mathrm{~nm}$; relative tolerance: $\left.10^{-5}\right)$ [28].

The trapped oil system shown in Fig. 1 is built in three stages. In stage I, a slab of oil (in contact with the left piston) and a slab of high salinity brine (in contact with the rock and $\sim 3 \mathrm{~nm}$ thick in the $x$-direction) are packed into the left reservoir. The rock pore and the right reservoir are filled with $2 \mathrm{M} \mathrm{NaCl}$ solution. The system is then equilibrated for $4 \mathrm{~ns}$ while applying a pressure of $41 \mathrm{MPa}$ on both pistons. In stage II, the pressure on the right piston is reduced to $1 \mathrm{MPa}$ to generate a pressure difference of $40 \mathrm{MPa}$ between the left and right reservoirs. Because this pressure difference is higher than the pore's capillary pressure $p_{c}=2 \sigma / W \approx 17.3 \mathrm{MPa}$ ( $W$ is the pore width, and the interfacial tension of the decane-brine interface is determined to be $52.24 \pm 1.07$ $\mathrm{mN} / \mathrm{m}$ in a separate equilibrium simulation), the oil invades into the pore, but a thin brine film remains on the pore's surface. This process is allowed to occur until a portion of the oil protrudes from the pore's right entrance in a droplet shape (see Fig. 1). Afterward, the left piston is held in space, and the oil in the left reservoir and the pore become trapped. In the following, $t=0$ is defined as the end of stage II. In stage III, the system is allowed to relax for $160 \mathrm{~ns}$, during which the number of water molecules and ions reaches an equilibrium.

To study the potential recovery of the trapped oil due to salinity reduction in the brine reservoir, at the end of the above stage III, all pairs of $\mathrm{Na}^{+}$and $\mathrm{Cl}^{-}$ions in the brine reservoir that are more than $1.5 \mathrm{~nm}$ away from the pore's entrance in the $x$-direction are removed. The calculation indicates that this reduces the average ion concentration in the brine reservoir to $\sim 0.1 \mathrm{M}$. The system is then run for $80 \mathrm{~ns}$. As a reference, the system with the high salinity in the brine reservoir is also run for $80 \mathrm{~ns}$. 
(a)
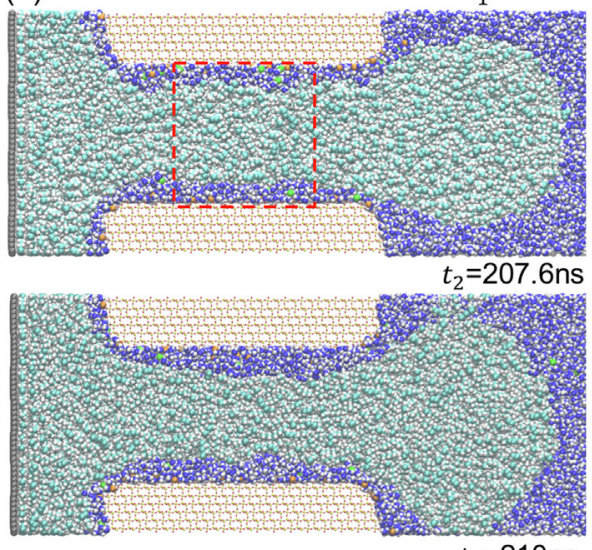

$t_{3}=210 \mathrm{~ns}$

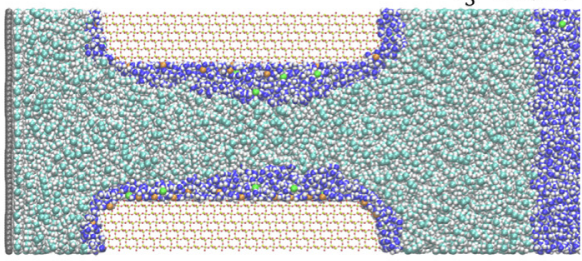

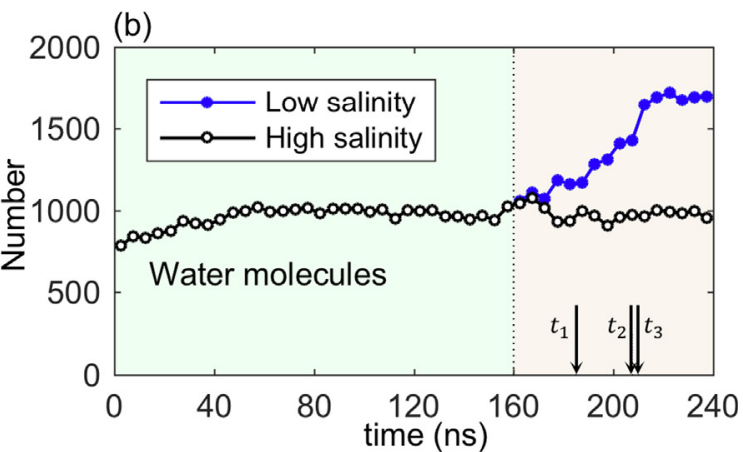

(c)

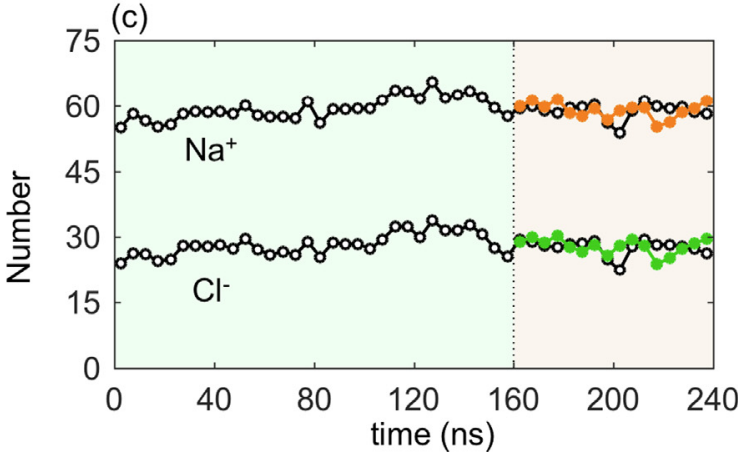

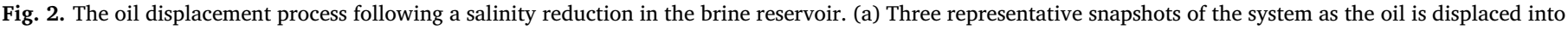

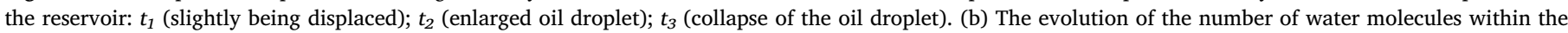

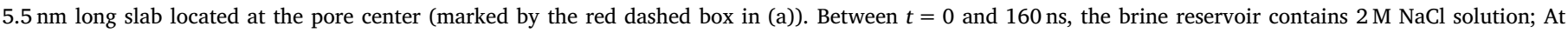

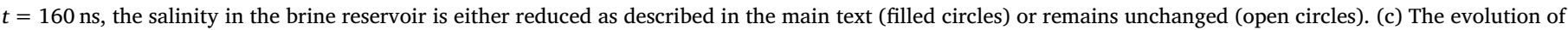

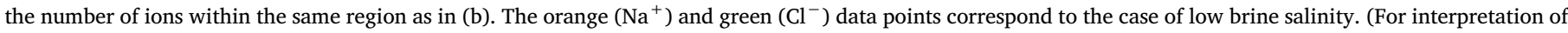
the references to color in this figure legend, the reader is referred to the web version of this article.)

\section{Results and discussions}

\subsection{Oil displacement}

Fig. 2a shows the snapshots of the system after the salinity of the brine reservoir is lowered abruptly at $t=160 \mathrm{~ns}$. Between 160 and $190 \mathrm{~ns}$, the system is essentially similar to the reference system where the salinity is not changed. For example, at $t_{1}=185 \mathrm{~ns}$, the oil droplet in Fig. 2a is similar to that of Fig. 1 under high salinity, and a thin brine film about $0.80 \mathrm{~nm}$ thick is observed on the pore walls. After that, water invades into the brine films, which forces the oil droplet protruding from the pore entrance to grow. As the droplet grows, eventually it touches its periodic images as shown in the snapshot at $t_{2}=207.6 \mathrm{~ns}$ (note that, as in most MD simulations, our system is periodic). At $t_{3}=210 \mathrm{~ns}$, the oil droplet collapses by merging with its periodic images (see Fig. S1 in the Supporting Information), and an oil layer appears in front of the pore. The system then reaches a new equilibrium, and this oil layer remains stable within the time scale $(\sim 100 \mathrm{~ns})$ explorable in MD simulations. For example, the distributions of water, oil, and ions near the film are nearly unchanged between the time frames 220-230 ns and 230-240 ns (see Fig. S2 in the Supporting Information).

The crucial observation of our simulation is that the oil is displaced out of the pore following a reduction of the salinity in the brine reservoir. In practice, the distance between neighboring pore throats is large. Therefore, each droplet protruding out of the pore typically grows independent of each other. When the droplet becomes large enough, it can be removed by external flow or thermal fluctuation (e.g., the thin neck within the pore can break through fluctuations, which leads to the release of oil droplets). In our simulations, as in most MD simulations, the system extends no more than tens of nanometers in any direction, and thus the distance between the droplet and its periodic image is small. Therefore, before the droplet is large enough to be removed by means similar to those in practice, it merges with its periodic images. This is undesirable but avoiding it requires a much larger system. For example, the simulation box size in $z$-direction needs to be at least doubled to $\sim 20 \mathrm{~nm}$ to eliminate the boundary issues, and about 200 computer cores must be used in the new simulations so that results are obtained in a reasonable amount of time. This will incur a prohibitive computational cost (even the present system took about three months to simulate).

Nevertheless, because the initial displacement of oil from the pore (and the associated oil droplet growth) is crucial for enhanced oil recovery, the results prior to droplet merging are relevant to practical systems. In addition, even though less frequently, merging of a droplet with its neighboring droplet(s) can occur in reality, especially in regions where the porosity and oil saturation are large. Below, we will focus on the initial displacement of oil from the nanopore following the salinity reduction in the brine reservoir.

To quantify the water invasion and oil displacement process, we compute the number of water molecules and ions in the thin brine film in the middle part of the pore (see the $5.5 \mathrm{~nm}$-wide red dashed box in Fig. 2a). The results are shown in Fig. $2 \mathrm{~b}$ and 2c. In the first $160 \mathrm{~ns}$ of simulation, the number of water molecules and ions in the brine film slowly increases, and an equilibrium is reached by $\sim 80 \mathrm{~ns}$. At equilibrium, the pressure of the oil in the nanopore is elevated due to the capillary effects (note the curvature of the droplet-shaped oil protruding from the pore is very large), and it is balanced by the disjoining pressure developed within the thin brine film. When the salinity in the brine reservoir is not altered, the number of water molecules and ions in the brine film fluctuates around their equilibrium values. When the salinity in the brine reservoir is reduced at $t=160 \mathrm{~ns}$ as described in Section 2, the number of water molecules in the film remains little changed for a few tens of nanoseconds. Afterward, it increases steadily until the oil droplet collapses at $t_{3}=210 \mathrm{~ns}$. These observations are consistent with the growth of the brine film shown in Fig. 2a. In sharp contrast, the number of ions within the brine film remains little 

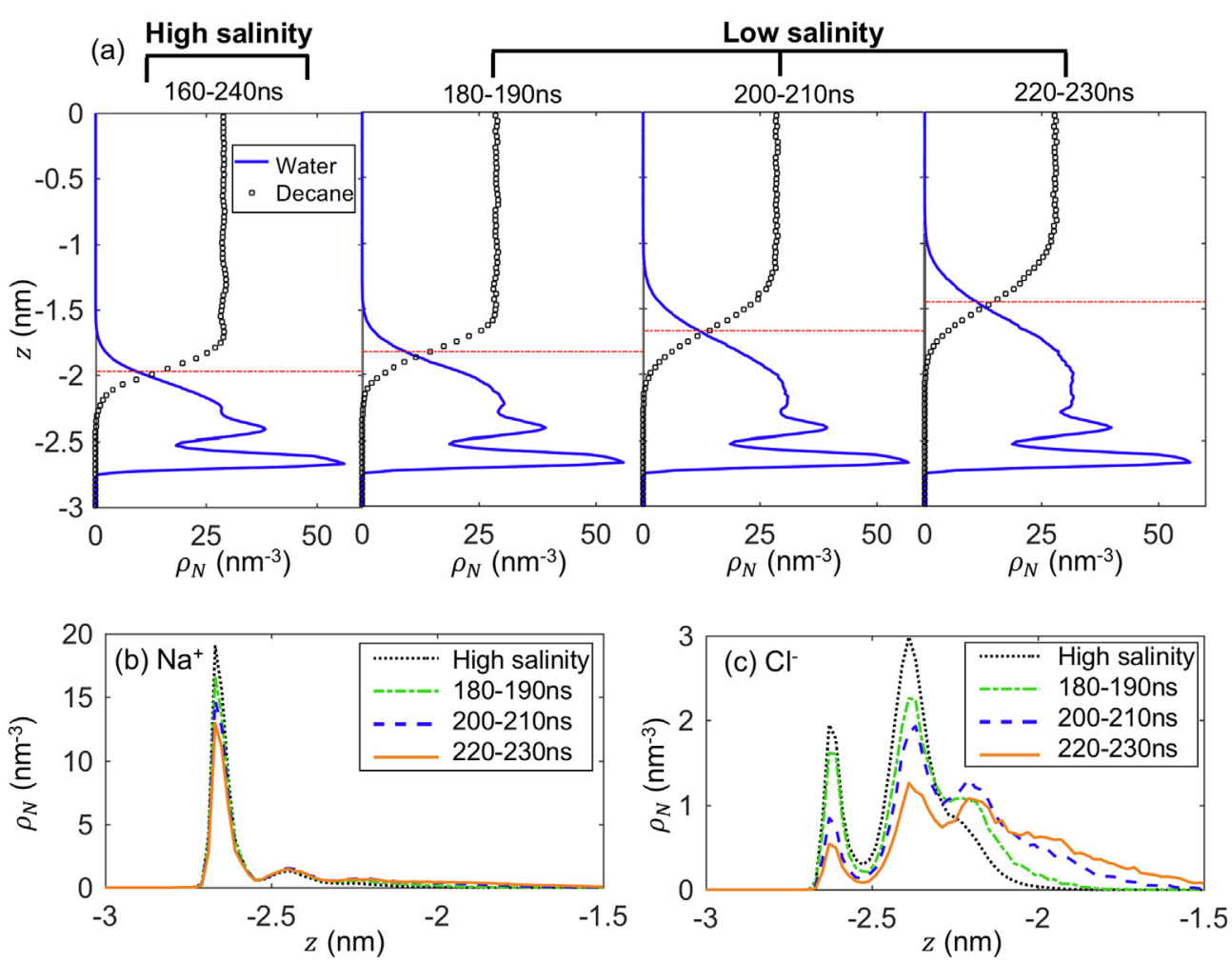

Fig. 3. The evolution of thin brine films on the pore walls. (a) The density profiles of the water molecules and carbon atoms of oil in the film in the $z$-direction. The zero plane of the quartz surface is located at $z=-3$ $\mathrm{nm}$. The red dash-dot line denotes the water-oil interface, where the decane density is half of its bulk value. (b, c) The density profiles of $\mathrm{Na}^{+}$ions (b) and $\mathrm{Cl}^{-}$ions (c) in the brine film at the same time frames as in (a). (For interpretation of the references to color in this figure legend, the reader is referred to the web version of this article.) changed after the salinity in the reservoir is reduced, which will be discussed in Section 3.4.

The expansion of the brine film sandwiched between the pore wall and oil after a salinity reduction in the brine reservoir is reminiscent of the growth of brine film due to double layer expansion, which has been suggested to lead to wettability alteration $[10,11]$. To understand the expansion of the brine film observed here and the possible role of electrical double layers (EDLs) in such expansion, below, we characterize the evolution of the structure of the brine films and the distribution of the ions in them following a salinity reduction.

\subsection{Evolution of the brine films}

Fig. 3a shows the density profiles of water molecules and oil near the lower wall of the slit pore $(z=-3 \mathrm{~nm}$ is the quartz surface's zero plane). The density data averaged in three representative time frames are examined: $t=180-190 \mathrm{~ns}$ (when the brine film starts to grow, see Fig. 2a), $t=200-210 \mathrm{~ns}$ (when the oil droplet collapses), and $t=220-230 \mathrm{~ns}$ (when the film becomes isolated). The density profiles for the case that the salinity of brine reservoir remains at $2 \mathrm{M}$ are also shown.

Two features can be observed as the brine films expand. First, near the pore surface, two water layers, corresponding to two density peaks, are observed. The first layer corresponds to the water molecules hydrating the strongly hydrophilic quartz surface, and the second layer corresponds to water molecules coordinated to the first water layer $[14,21]$. Because of the strong quartz-water and water-water interactions, these two layers are little affected by the oil layer above the brine film, in agreement with our systematic study of the brine film structure in OBR systems [14]. Second, as a brine film expands, the brine-oil interface is pushed toward the pore's central line and the water distribution changes only moderately. The newly added water molecules mainly appear as bulk water. This is evident from the density profile during $t=220-230 \mathrm{~ns}$, showing a plateau adjacent to the second water peak whose height is close to the bulk water density. If we assign the location of the brine-oil interface to the position where the decane density is $50 \%$ of its bulk value (marked as red dashed lines in Fig. 3a), the thickness of the brine film is found to grow by $\sim 0.5 \mathrm{~nm}$ after the salinity in the brine reservoir is reduced.

Fig. 2c shows that the total number of ions within the brine film remains almost unchanged within $80 \mathrm{~ns}$ after the salinity in the brine reservoir is reduced. However, because the film expands, the ion distribution within the brine film does change, and the EDLs in the brine film expand. Fig. 3b shows the evolution of the $\mathrm{Na}^{+}$density profiles across the brine film after the salinity is reduced in the reservoir. At high salinity, two peaks corresponding to $\mathrm{Na}^{+}$ions contact adsorbed on the quartz surface and $\mathrm{Na}^{+}$ions that try to maximize their hydration by the interfacial water are observed as in our recent work on EDLs in brine films [14]. As the salinity is reduced and the brine film expands, the first $\mathrm{Na}^{+}$peak becomes lower, and the desorbed $\mathrm{Na}^{+}$ions move into the region $\mathrm{z}>-0.23 \mathrm{~nm}$. This is caused by the fact that, as the brine films grow, the brine-oil interface recedes toward the pore's centerline and thus the $\mathrm{Na}^{+}$ions in this region become better hydrated.

Fig. 3c shows the evolution of the density profiles of the $\mathrm{Cl}^{-}$ions following the reduction of salinity in the brine reservoir. Under high salinity, two peaks of $\mathrm{Cl}^{-}$ions appear next to the first and second $\mathrm{Na}^{+}$ peaks. This is likely caused by the enhanced pairing of $\mathrm{Na}^{+}$and $\mathrm{Cl}^{-}$ ions in thin brine films. As shown recently [14], the effective dielectric constant of water in thin films is smaller than their bulk value and decreases as the thin film thickness decreases. For example, in a $0.72 \mathrm{~nm}$-thick film, the dielectric constant is only $57 \%$ of the bulk value. The reduced dielectric screening prompts the pairing of $\mathrm{Na}^{+}$and $\mathrm{Cl}^{-}$ions, thus leading to the two peaks observed in Fig. 3c. When the salinity is reduced, and the brine film expands, the dielectric screening ability of the water in the brine film improves, and thus the ion pairing and the $\mathrm{Cl}^{-}$peaks adjacent to the $\mathrm{Na}^{+}$peaks are reduced. The emergence of the second $\mathrm{Cl}^{-}$peak at $0.8 \mathrm{~nm}$ from the quartz surface is similar to that observed near negatively charged walls in contact with $\mathrm{NaCl}$ solution and is caused by the more favorable hydration of the $\mathrm{Cl}^{-}$ ions at this position [29]. Overall, following the reduction of the salinity of the brine reservoir, the brine film expands, the disparity of both $\mathrm{Na}^{+}$and $\mathrm{Cl}^{-}$densities across the film becomes weaker. 

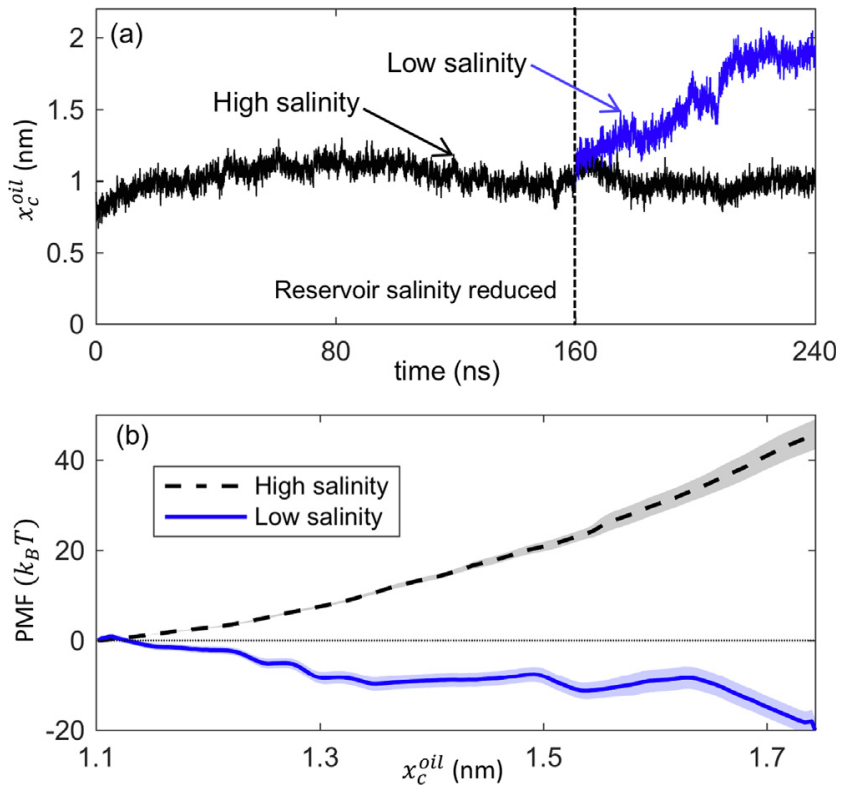

Fig. 4. Oil displacement and the associated PMF. (a) The evolution of the center-of-mass position of the entire oil phase with respect to the pore center in the $x$-direction $\left(x_{c}^{\text {oil }}\right)$ at high and low brine reservoir salinity computed in nonequilibrium simulations. (b) The PMF profiles of oil displacement as a function of $x_{c}^{\text {oil }}$ under high and low brine salinity computed using umbrella sampling in quasi-equilibrium simulations. The errors of the PMF profiles are represented by the shaded areas.

\subsection{Thermodynamics of the oil displacement process}

To understand the thermodynamics of the oil displacement following the salinity reduction in the brine reservoir, we compute the potential of mean force (PMF) profile as oil is displaced from the pore. The reaction coordinate for the oil displacement is taken as the center of mass position of the oil phase in the $x$-direction, $x_{c}^{\text {oil }}$. As shown in Fig. 4a, after the brine reservoir's salinity is reduced, $x_{c}^{\text {oil }}$ moves toward the brine reservoir by $\sim 0.85 \mathrm{~nm}$, whereas $x_{c}^{\text {oil }}$ changes little when the brine reservoir's salinity is unchanged. Therefore, we compute the PMF when $x_{c}^{\text {oil }}$ of the oil moves from its equilibrium position at high reservoir salinity toward the brine reservoir by $\sim 0.8 \mathrm{~nm}$.

The PMF as a function of $x_{c}^{\text {oil }}$ is obtained in a separate series of quasi-equilibrium simulations using the umbrella sampling technique [30]. A set of initial configurations of the system with a window size of $0.05 \mathrm{~nm}$ are generated by pulling the oil phase in $x$-direction at a constant speed of $0.25 \mathrm{~nm} / \mathrm{ns}$. Subsequent umbrella sampling runs are performed on each system by relaxing the system first for $10 \mathrm{~ns}$ and then performing a $30 \mathrm{~ns}$ run. In these simulations, a force is applied to each atom of the decane molecules to constrain the oil's center-of-mass position around the center of individual sampling window. This force is given by $F_{\text {com }}=K\left(x_{c}^{\text {oil }}-\zeta\right)^{2}$, where $x_{c}^{\text {oil }}$ and $\zeta$ are the oil's center-ofmass position and the center of each sampling window in the $x$-direction, respectively. $K$ is a spring constant taken as $50000 \mathrm{~kJ} /\left(\mathrm{mol} \cdot \mathrm{nm}^{2}\right)$. The PMF is next obtained from the histogram of the center of mass position of the oil in each window using the WHAM method [31]. The error bar of the PMF is evaluated using 100 bootstrap samples.

Fig. 4b shows the PMF profiles for oil displacement for the cases when the brine reservoir salinity is unchanged (i.e., $2 \mathrm{M}$ ) and reduced, as discussed in Section 2. For the case where the brine reservoir salinity remains high, the PMF increases as oil is displaced, i.e., the displacement of oil into the brine reservoir is thermodynamically unfavorable. The increasing PMF with the oil displacement is dominated by the energy of the brine-oil interfaces. Because these interfaces' area increases nonlinearly with the reaction coordinate $x_{c}^{\text {oil }}$, PMF grows faster as $x_{c}^{\text {oil }}$ increases. The significant growth of PMF with $x_{c}^{\text {oil }}$ is consistent with the fact that the oil remains trapped by the nanopore in this case. In contrast, for the case where the brine reservoir salinity is reduced, the PMF decreases as oil is displaced into the brine reservoir, which agrees well with the spontaneous oil displacement shown in Fig. 2. The different PMFs for these cases can be understood as follows.

As the oil is displaced from the pore, the PMF is affected by two competing processes. On the one hand, the displacement of oil increases the oil-brine interfacial area (see Fig. 2a), which tends to increase the PMF. On the other hand, as the oil is displaced from the pore, the thickness of the brine film on the pore surface increases. Because the disjoining pressure within the brine film is repulsive, the thickening of the brine film tends to reduce the free energy associated with it and thus the total PMF. For the high salinity case, the disjoining pressure is small. Thus the reduction of the thin brine film energy is overwhelmed by the increase of the oil-brine energy, and the PMF increases as oil is displaced. For the low salinity case, the disjoining pressure due to the EDL forces is much larger [32]. Therefore, as oil is displaced from the pore, the reduction of brine film's energy dominates over the increase of the oil-brine interfacial energy, leading to a decrease of the PMF.

The favorable displacement of oil from the pore at low salinity can also be understood from the perspective of water. Initially, the water molecules in the brine film are in equilibrium with the brine reservoir. After the salinity of the brine reservoir is reduced, the chemical potential of the water molecules in the brine reservoir becomes much higher than those in the brine film, which has higher ion concentration. Therefore, the water molecules in the brine reservoir spontaneously move into the brine film by osmosis, and the oil is displaced from the pore at the same time. It is worth noting that this interpretation is also in line with the interpretation from the perspective of disjoining pressure because the EDL disjoining pressure is dominated by osmotic effects $[14,32,33]$.

For the low salinity case, in addition to the overall decrease of the $\mathrm{PMF}$ as $x_{c}^{\text {oil }}$ increases, some finer features are also observed, e.g., several weak plateaus are observed for $x_{c}^{\text {oil }}$ between $\sim 1.25$ and $1.63 \mathrm{~nm}$. These plateaus are likely caused by the discreteness of water and ion layers in the brine films. As shown in Fig. 3, on average, there are 2 to 3 layers of water and ions in the brine films. However, locally along the pore length, brine films can be in metastable states with either 2 or 3 layers of water/ion. As $x_{c}^{\text {oil }}$ increases (i.e., the oil is displaced from pore or as water enters the brine film within the pore), these local metastable states can persist for a while before abruptly disappear, which leads to the plateaus and step-like changes in the PMF curve. In addition, the difficulties in sampling may also cause these apparent plateaus, e.g., the statistical errors are more pronounced in the plateau regions (especially for $x \sim 1.52-1.63 \mathrm{~nm}$ ).

\subsection{Dynamics of the ions in brine films}

An interesting aspect of the brine film expansion observed in Fig. 2a is that the number of ions within the brine film barely decreases within $80 \mathrm{~ns}$ after the salinity of the brine reservoir is reduced. This differs from the usual expectation that the ions readily diffuse out the brine film upon a reduction of the brine reservoir's salinity.

The very slow depletion of ions from the brine film is partly caused by the slow diffusion of the ions within the brine film. Fig. 5a and $5 \mathrm{~b}$ show the mean square displacement (MSD) of the ions in the film within the $x y$-plane. The MSD curves of ions in a bulk brine at $2 \mathrm{M}$ are also obtained from a separate equilibrium simulation. The self-diffusion coefficients of ions are evaluated via the Einstein relation: $D=\lim _{t \rightarrow \infty}\left\langle\left(x_{i}(t)-x_{i}(0)\right)^{2}+\left(y_{i}(t)-y_{i}(0)\right)^{2}\right\rangle / 4 t$ for the ions in brine films and $\stackrel{t \rightarrow \infty}{=} \lim _{t \rightarrow \infty}\left\langle\left(x_{i}(t)-x_{i}(0)\right)^{2}+\left(y_{i}(t)-y_{i}(0)\right)^{2}+\left(z_{i}(t)-z_{i}(0)\right)^{2}\right\rangle / 6 t$ for the ions in bulk (see the fitted solid lines in Fig. $5 \mathrm{a}$ and b). For ions in brine films, their diffusion coefficients are found to be $D_{\mathrm{Na}^{+}}^{\text {film }}=0.054 \times 10^{-9} \mathrm{~m}^{2} / \mathrm{s}$ and $D_{\mathrm{Cl}^{-}}^{\text {film }}=0.293 \times 10^{-9} \mathrm{~m}^{2} / \mathrm{s}$. For ions in bulk brine, we obtain $\quad D_{\mathrm{Na}^{+}}^{\text {bulk }}=1.938 \times 10^{-9} \mathrm{~m}^{2} / \mathrm{s} \quad$ and 

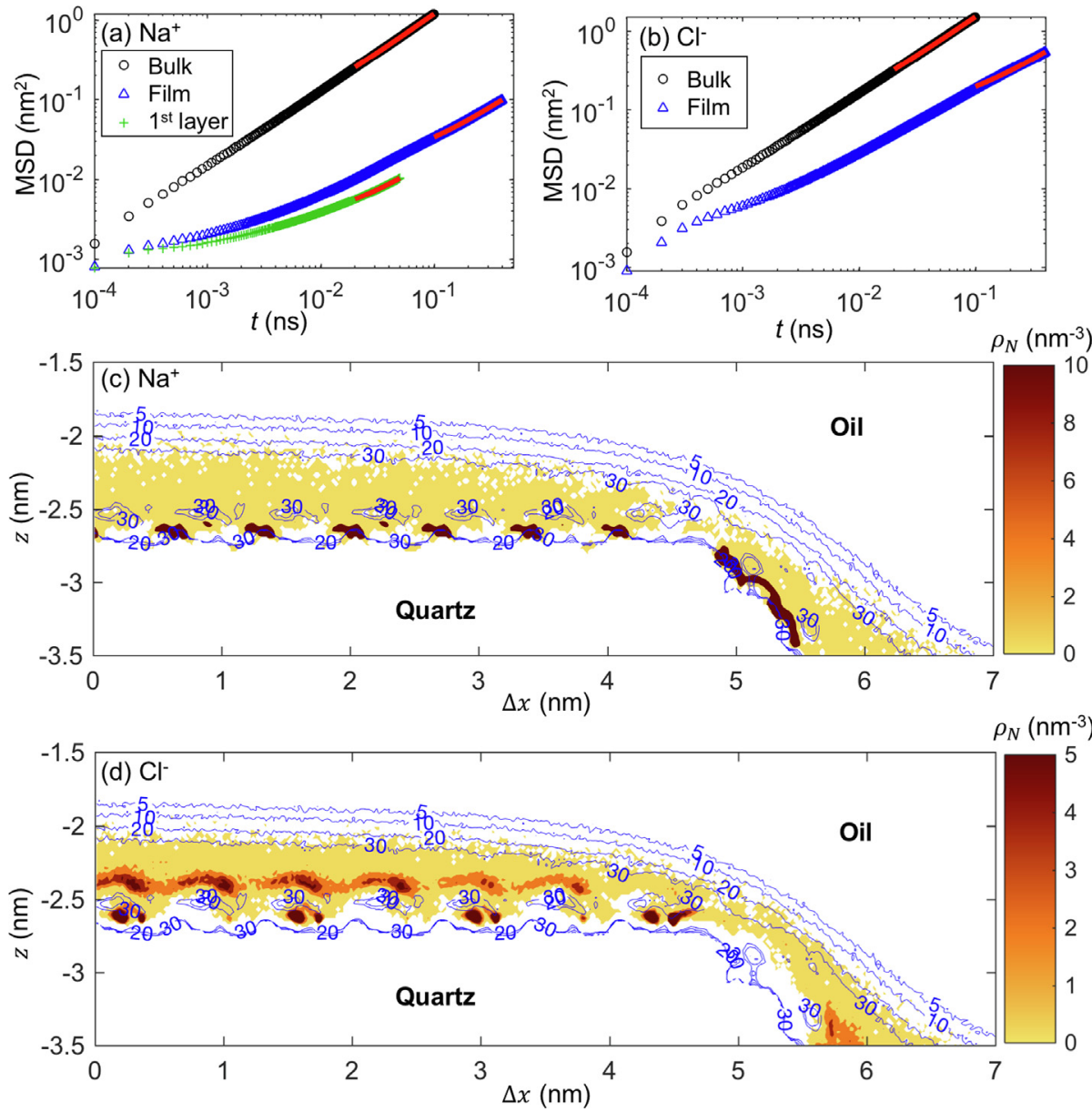

Fig. 5. The dynamics of ions in thin brine films. (a) The mean square displacement (MSD) of $\mathrm{Na}^{+}$ions in bulk $2 \mathrm{M}$ brine, across the entire brine film (cf. the dashed box in Fig. 2), and within the inner layer (cf. $\mathrm{z}<-2.55 \mathrm{~nm}$ in Fig. 3b) at high salinity. (b) The MSD of $\mathrm{Cl}^{-}$ions in the same bulk brine and within the same film as in (a). The MSDs are fitted to the solid red line to obtain the diffusion coefficients. (c) The density of the $\mathrm{Na}^{+}$ions (color coded) overlapped on the density of water (shown as blue contour lines) near the quartz surface in the $x z$-plane. The pore's geometric center is located at $x=0$ and $z=0$. (d) The distribution of $\mathrm{Cl}^{-}$ions and water with the same setup as in (c). (For interpretation of the references to color in this figure legend, the reader is referred to the web version of this article.)
$D_{\mathrm{Cl}^{\text {bulk }}}^{\text {bu }}=2.481 \times 10^{-9} \mathrm{~m}^{2} / \mathrm{s}$. Therefore, the diffusion of the $\mathrm{Na}^{+}$and $\mathrm{Cl}^{-}$ ions confined in the brine films in the high salinity system is 36 and 8 times slower than that of bulk $\mathrm{Na}^{+}$and $\mathrm{Cl}^{-}$ions, respectively. These slow dynamics of ions are responsible for the negligible ion depletion following the salinity reduction of the brine reservoir. The slowdown of ion diffusion is likely caused by the viscoelectric effect [34]. This effect is closely related to the modification of water molecules' orientation by highly charged solid surfaces and can slow down the diffusion of ions adjacent to these surfaces [34]. In our simulations, we divide the $\mathrm{Na}^{+}$ ions in the brine film into an inner layer $(\mathrm{z}<-2.55 \mathrm{~nm}$ in Fig. 2b) and an outer layer $(\mathrm{z}>-2.55 \mathrm{~nm})$. The inner layer $\mathrm{Na}^{+}$ions account for $\sim 81 \%$ of $\mathrm{Na}^{+}$ions in the brine film and have a diffusion coefficient of $0.04 \times 10^{-9} \mathrm{~m}^{2} / \mathrm{s}$ (fitted from the MSD within 1 st $\mathrm{Na}^{+}$layer in Fig. 5a). Hence, the diffusion of $\mathrm{Na}^{+}$ions is slowest in the inner layer, which is consistent with the expectations based on the viscoelectric effect.

Another factor contributing to the slow ion depletion from brine films is the hindrance caused by the edge at pore entrance. Fig. $5 \mathrm{c}$ and $\mathrm{d}$ show the color-coded ion density overlapped on the water density (shown in blue contour lines) in the $x z$-plane. These data are obtained from the high salinity system from 160 to $240 \mathrm{~ns}$. Away from the pore's edge $(x<3 \mathrm{~nm}), \mathrm{Na}^{+}$ions accumulate mainly next to the quartz surface but can also access space $\sim 0.4 \mathrm{~nm}$ from the quartz surface, which is consistent with ion density profiles shown in Fig. $3 c$ and d. Near the pore's edge ( $4 \mathrm{~nm}<x<5 \mathrm{~nm}$ ), although $\mathrm{Na}^{+}$ions are still found next to the quartz surface, the space they can access adjacent to the quartz surface becomes considerably narrower. The reduced access of $\mathrm{Na}^{+}$ ions to the space near the pore's edge is expected: near the pore's edge, the brine-oil interface has a large curvature and higher capillary pressure, and the latter drives the brine film thinner [35]; as the brine film is thinned, the hydration of the $\mathrm{Na}^{+}$ions in the film worsens, which hinders the access of $\mathrm{Na}^{+}$ions to the space near the pore's edge and the diffusion of $\mathrm{Na}^{+}$ions out of the brine film. The reduced access of the space near the pore edge also applies to the $\mathrm{Cl}^{-}$ions (see Fig. 5d), and thus the above hindrance effect also occurs for the diffusion of $\mathrm{Cl}^{-}$ions out of the pore.

Although the depletion of ions from brine films is extremely slow in the present system, it does not contradict the displacement of oil from the pore observed in Fig. 2. Once the salinity in the brine reservoir is reduced, the chemical potential of water inside the reservoir becomes higher than that in the brine film. Therefore, the osmotic pressure that drives water molecules into the pore is developed, and the influx of water molecules into the pore displaces oil from the pore. Nevertheless, we note that the displacement of oil following the present mechanism is a rather slow process: the transport of water into the pore experiences large resistance as it occurs through molecularly thin brine films. This mechanism may contribute to the slow oil recovery observed in previous LSW experiments performed in micromodels of OBR systems [9].

\section{Conclusions}

In summary, we report an MD study of the recovery of oil trapped by nanopores. Lowering the brine salinity in the reservoir promotes the expansion of the thin brine film on the rock surface; subsequently, the displacement of oil out of the nanopore. An oil droplet protruding from the pore entrance into the brine reservoir eventually collapses by merging with nearby oil droplets (in our simulations, its periodic images). The expansion of brine films is critical for the oil displacement, and it occurs through the osmosis of water molecules from the brine reservoir. In the present study, as the brine films expand, ions barely leave the film even though the EDLs in the films expand due to the 
increase of film thickness. The slow depletion of ions from the brine films is caused by the slow diffusion of ions in brine films (roughly one order of magnitude slower than in bulk brine) and the narrow ion transport pathway near the edge of pore entrances. Since the osmosis of water into the pore occurs through thin brine films is slow, it may help explain the anomalously slow recovery of oil reported in recent micromodeling experiments of LSW.

As a first study, this work focuses on a relatively simple system. More realistic systems, e.g., those featuring divalent ions in the brine, nested nanopores, and rock substrates with different compositions and surface charge densities, can be studied in the future to understand better the enhanced recovery of oil trapped by pores. Nevertheless, following a reduction of the salinity of the brine reservoir, the transport of water into the pores by osmosis and the subsequent displacement of oil from the pore, both highly robust phenomena that do not depend sensitively on the composition of brine and rock, should still play a key role in the oil recovery in those more complicated systems.

\section{Declaration of Competing Interest}

The authors declare that they have no known competing financial interests or personal relationships that could have appeared to influence the work reported in this paper.

\section{Acknowledgments}

We thank the ARC at Virginia Tech for generous allocations of computer time. R. Q. acknowledges the financial support of the NSF under grant CBET 1705287. S.S. acknowledges the financial support of King Abdullah University of Science and Technology (KAUST) through the grants BAS/1/1351-01 and URF/1/2993-01.

\section{Appendix A. Supplementary data}

Supplementary data to this article can be found online at https:// doi.org/10.1016/j.fuel.2019.116443.

\section{References}

[1] Muggeridge A, Cockin A, Webb K, Frampton H, Collins I, Moulds T, et al. Recovery rates, enhanced oil recovery and technological limits. Philos Trans R Soc A: Math, Phys Eng Sci 2014:372:20120320.

[2] Bartels W-B, Mahani H, Berg S, Hassanizadeh S. Literature review of low salinity waterflooding from a length and time scale perspective. Fuel 2019;236:338-53.

[3] RezaeiDoust A, Puntervold T, Strand S, Austad T. Smart water as wettability modifier in carbonate and sandstone: a discussion of similarities/differences in the chemical mechanisms. Energy Fuels 2009;23:4479-85.

[4] Katende A, Sagala F. A critical review of low salinity water flooding: mechanism, laboratory and field application. J. Mol. Liq. 2019.

[5] Sheng J. Critical review of low-salinity waterflooding. J Petrol Sci Eng 2014;120:216-24.

[6] Austad T, RezaeiDoust A, Puntervold T. In Chemical Mechanism of Low Salinity Water Flooding in Sandstone Reservoirs, SPE improved oil recovery symposium, Society of Petroleum Engineers: 2010.

[7] Qiao C, Johns R, Li L. Modeling low-salinity waterflooding in chalk and limestone reservoirs. Energy Fuels 2016;30:884-95.
[8] Jerauld GR, Webb KJ, Lin C-Y, Seccombe J. In Modeling Low-Salinity Waterflooding, SPE Annual Technical Conference and Exhibition, Society of Petroleum Engineers: 2006.

[9] Mahani H, Berg S, Ilic D, Bartels W-B, Joekar-Niasar V. Kinetics of Low-SalinityFlooding Effect. Spe J 2015;20:8-20.

[10] Myint PC, Firoozabadi A. Thin liquid films in improved oil recovery from lowsalinity brine. Curr Opin Colloid Interface Sci 2015;20:105-14.

[11] Tian H, Wang M. Electrokinetic mechanism of wettability alternation at oil-waterrock interface. Surf Sci Rep 2017;72:369-91.

[12] Tian H, Wang M. Molecular dynamics for ion-tuned wettability in oil/brine/rock systems. AIP Adv 2017;7:125017.

[13] Xu S, Wang J, Wu J, Liu Q, Sun C, Bai B. Oil contact angles in a water-decane-silicon dioxide system: effects of surface charge. Nanoscale Res Lett 2018;13:108.

[14] Fang C, Sun S, Qiao R. Structure, thermodynamics, and dynamics of thin brine films in oil-brine-rock systems. Langmuir 2019;35:10341-53.

[15] Jiménez-Ángeles F, Firoozabadi A. Contact angle, liquid film, and liquid-liquid and liquid-solid interfaces in model oil-brine-substrate systems. J Phys Chem C 2016;120:11910-7.

[16] Zhao J, Yao G, Ramisetti SB, Hammond RB, Wen D. Molecular dynamics investigation of substrate wettability alteration and oil transport in a calcite nanopore. Fuel 2019;239:1149-61.

[17] Ligthelm DJ, Gronsveld J, Hofman J, Brussee N, Marcelis F, van der Linde H. In Novel Waterflooding Strategy by Manipulation of Injection Brine Composition, EUROPEC/EAGE conference and exhibition, Society of Petroleum Engineers: 2009.

[18] Berendsen H, Grigera J, Straatsma T. The missing term in effective pair potentials. J Phys Chem 1987;91:6269-71.

[19] Siu SW, Pluhackova K, Böckmann RA. Optimization of the Opls-Aa Force Field for Long Hydrocarbons. J Chem Theory Comput 2012;8:1459-70.

[20] Joung IS, Cheatham III TE. Determination of alkali and halide monovalent ion parameters for use in explicitly solvated biomolecular simulations. J Phys Chem B 2008;112:9020-41.

[21] Kroutil O, Chval Z, Skelton A, Predota M. Computer Simulations of Quartz (101)-Water Interface over a Range of Ph Values. J Phys Chem C 2015;119:9274-86.

[22] Emami FS, Puddu V, Berry RJ, Varshney V, Patwardhan SV, Perry CC, et al. Force field and a surface model database for silica to simulate interfacial properties in atomic resolution. Chem Mater 2014;26:2647-58.

[23] Lee SY, Webb KJ, Collins I, Lager A, Clarke S, O‘Sullivan M, Routh A. In Low Salinity Oil Recovery-Increasing Understanding of the Underlying Mechanisms of Double Layer Expansion, IOR 2011-16th European symposium on improved oil recovery, 2011.

[24] Buckley J, Liu Y. Some mechanisms of crude oil/brine/solid interactions. J Petrol Sci Eng 1998;20:155-60.

[25] Cygan RT, Liang J-J, Kalinichev AG. Molecular models of hydroxide, oxyhydroxide, and clay phases and the development of a general force field. J Phys Chem B 2004;108:1255-66.

[26] Abraham MJ, Murtola T, Schulz R, Páll S, Smith JC, Hess B, et al. Gromacs: high performance molecular simulations through multi-level parallelism from laptops to supercomputers. SoftwareX 2015;1:19-25.

[27] Bussi G, Donadio D, Parrinello M. Canonical Sampling through Velocity Rescaling. J Chem Phys 2007;126:014101.

[28] Darden T, York D, Pedersen L. Particle mesh ewald: An N. Log (N) method for ewald sums in large systems. J Chem Phys 1993;98:10089-92.

[29] Qiao R, Aluru NR. Scaling of electrokinetic transport in nanometer channels. Lanomuir 2005;21:8972-7.

[30] Torrie GM, Valleau JP. Nonphysical sampling distributions in monte carlo freeenergy estimation: umbrella sampling. J Comput Phys 1977;23:187-99.

[31] Kumar S, Rosenberg JM, Bouzida D, Swendsen RH, Kollman PA. The weighted histogram analysis method for free-energy calculations on biomolecules. I. The method. J Comput Chem 1992;13:1011-21.

[32] Israelachvili JN. Intermolecular and Surface Forces. Academic press; 2015.

[33] Schlaich A, dos Santos AP, Netz RR. Simulations of nanoseparated charged surfaces reveal charge-induced water reorientation and nonadditivity of hydration and mean-field electrostatic repulsion. Langmuir 2018;35:551-60.

[34] Hsu W-L, Harvie DJ, Davidson MR, Dunstan DE, Hwang J, Daiguji H. Viscoelectric effects in nanochannel electrokinetics. J. Phys. Chem. C 2017;121:20517-23.

[35] Hu H, Weinberger CR, Sun Y. Effect of nanostructures on the meniscus shape and disjoining pressure of ultrathin liquid film. Nano Lett. 2014;14:7131-7. 